\title{
Effective chiral lagrangian from QCD at nonzero chemical potential
}

\author{
R.F. Alvarez-Estrada, A. Gómez Nicola \\ Departamento de Física Teónica, Universidad Complutense, 28040, Madrid, Spain \\ Received 23 May 1995 \\ Editor: R. Gatto
}

\begin{abstract}
We start from the euclidean QCD action for gluons and massless quarks with $N_{c}$ colours at finite baryon chemical potential $\mu_{B}$ and zero temperature. For $\mu_{B}$ of the order of external momenta $o(p)$ we derive an euclidean effective real chiral lagrangian at finite $\mu_{B}$, up to and including $o\left(p^{4}\right)$, in terms of Goldstone Bosons (GB), in the large $N_{c}$ limit, including gluon contributions. Our effective action generalizes non-trivially the one obtained for $\mu_{B}=0$ by previous authors, and it includes new $\mu_{B}$-dependent terms. In particular, a topological term $\mu_{B} N_{B}$ is found, $N_{B}$ being the baryon number in terms of GB fields with the correct normalization factor. Physical implications of the remaining $\mu_{B}$-dependent terms are discussed briefly.
\end{abstract}

In recent years, the low-energy dynamics of QCD has been extensively studied in the framework of chiral effective lagrangians. In the first works [1], in which chiral perturbation theory $(\chi \mathrm{PT})$ was applied successfully, the most general effective lagrangian displaying all the symmetries required, and containing only Goldstone Bosons (GB) and external sources, was derived up to order $o\left(p^{4}\right)$ in external momenta and quark masses. Another approach also aimed at obtaining the effective lagrangian starts directly from QCD [2-5] and assumes that the number of colours $\left(N_{c}\right)$ is large. We will make use of this method below.

The effective lagrangian and $\chi \mathrm{PT}$ programs have also been extended so as to include finite temperature effects. Thermodynamical properties of a hadronic medium in thermal equilibrium have been studied at zero density in $[6,7]$. In this paper, we shall extend this program for the case of a medium at finite density by including the baryon chemical potential in the
QCD lagrangian. Previous studies of chiral matter at finite temperature and density can be found in [8$10]$. We will perform our analysis for the $T=0$ case. Our starting point will be the QCD partition function for massless quarks (chiral limit) at nonzero chemical potential, which is given in euclidean space-time [11-13] by

$$
\begin{aligned}
& Z\left[\mu_{B}\right]=\int[\tilde{d G}][d \bar{q}][d q] \\
& \quad \times \exp -\int d^{4} x\left[\mathcal{L}-\bar{q} \frac{\mu_{B}}{N_{c}} I y_{0} q\right]
\end{aligned}
$$

where $q$ are the unrenormalized quark fields represented by a column of $N_{f}$ Dirac spinors, $N_{f}$ being the number of flavours. The gluonic measure $[\hat{d} G]$ incorporates the Faddeev-Popov determinant and the YangMills piece $\exp \left(-\frac{1}{4} G_{\mu \nu}^{a} G_{\mu \nu}^{a}\right)$. We have $\mathcal{L}=\bar{q} \gamma_{\mu z} \Delta_{\mu} q$ with $\Delta_{\mu}=\partial_{\mu}+\frac{i g}{2} \lambda^{a} G_{\mu}^{a}, G_{\mu}^{a}$ being the unrenormalized 
gluon fields, and $G_{\mu \nu}^{a}=\partial_{\mu} G_{\nu}^{a}-\partial_{\nu} G_{\mu}^{a}-g f^{a b c} G_{\mu}^{b} G_{\nu}^{c}$ with $\lambda^{a}$ the generators of $S U\left(N_{c}\right)$ and $f^{a b c}$ its structure constants. We shall use euclidean gamma matrices $\gamma_{\mu}$ and $\gamma_{5}$, which are hermitian, with $\left\{\gamma_{\mu}, \gamma_{\nu}\right\}=2 \delta_{\mu \nu}$ and $\left\{\gamma_{\mu}, \gamma_{5}\right\}=0$. Overall normalization constants in path integrals will be included in the gluonic measure and will never be written explicitly. In Eq. (1) we have restricted ourselves to the case of a chemical potential term $\frac{\mu_{B}}{N_{c}} l$, attributing the same chemical potential to each flavour, which reflects baryon number conservation in QCD. Thus, $I$ is the unit $N_{f} \times N_{f}$ matrix and $\mu_{B} \neq 0$ is the baryon chemical potential. Physically, this corresponds to a medium at finite baryon number density.

We are interested in deriving an effective lagrangian at finite density in terms of only GB fields, starting from (1). This lagrangian would then lead to an effective theory, valid at low energies. To be consistent, we will treat $\mu_{B}$ as a quantity of order $o(p)$, that is, of the same order as the external momentum appearing in the theory. Our effective lagrangian will then be valid in the low density regime, with respect to some energy scalc. We will be more precise about the order of magnitude of this scale below. The partition function in Eq. (1) could also be written as a path integral (not over quark and gluon fields but over GB fields) which, in turn, would contain an effective action depending also on GB fields. This program can be implemented up to a given momentum order, using $\chi \mathrm{PT}$, in terms of chiral loops, as it was done for low temperatures and $\mu_{B}=0$ in [7]. In order to obtain the effective lagrangian from QCD we shall need an important approximation, namely, we shall work in the large $N_{c}$ limit in which chiral loops are down by powers of $1 / N_{c}$. This is the approach followed in [2-4] for deriving the non-anomalous part of the effective lagrangian. In [3] and [5], this program has been extended to the anomalous part, that is, the WessZumino-Witten (WZW) action $[14,15]$. To be precise, we parametrize the GB field in the usual way as $U(x)=\exp \left(i \pi^{a} \lambda_{a} / f\right)$ with $U(x) \in S U\left(N_{f}\right)$. For instance, for $N_{f}=2, \pi^{a}$ and $f$ are the pion fields and the pion decay constant $f_{\pi}$, respectively. In the large $N_{c}$ limit the $U(x)$ field can be regarded as a classical field, since chiral loops are neglected. Furthermore, in order to derive the Green functions with vector and axial-vector current insertions, we should incorporate the corresponding sources $v_{\mu}(x), a_{\mu}(x)$ into the QCD lagrangian when building up the generating functional. These $v=\left(v_{\mu}\right), a=\left(a_{\mu}\right)$ fields can also be considered as gauge fields, thereby gauging the symmetry $S U_{L}\left(N_{f}\right) \times S U_{R}\left(N_{f}\right)$. Specifically, the sources $v_{\mu}(x)$ and $a_{\mu}(x)$ are traceless antihermitian matrices of $S U\left(N_{f}\right)$. In the non-anomalous effective action this will imply that ordinary derivatives acting on $U$ fields will be replaced by covariant ones. Thus, by consistency, the $v, a$ fields are $o(p)$ quantities, as it happens with ordinary derivatives acting on GB fields. A term depending on $U(x)$ and some mass parameter $M$ will also be included [4,5]. This is the parameter that sets the scale of the theory and allows us to perform the derivative expansion. This $M$-dependent term can be regarded as a constituent quark mass term [16], or simply as the linear sigma model coupling between quarks and GB fields. The effective action $\Gamma$ to be built up in this way will be a finite density generalization of the one obtained in $[1,4]$. Then, we will study the following euclidean effective action:

$$
\begin{aligned}
\exp & -\Gamma\left[\mu_{B}, v, a, U\right] \\
& =\int[\tilde{d} G][d \bar{q}][d q] \exp -S_{E}\left[\bar{q}, q, G, v, a, U, \mu_{B}\right] \\
& =\int[\tilde{d} G] \exp -\Gamma_{q}\left[G, v, a, U, \mu_{B}\right] \\
S_{E} & =\int d^{4} x\left[\mathcal{L}+\bar{q} \gamma_{\mu}\left(v_{\mu}(x)+\gamma_{5} a_{\mu}(x)-b_{\mu}\right) q\right. \\
& \left.+M\left(\bar{q}_{R} U(x) q_{L}+\bar{q}_{L} U^{\dagger}(x) q_{R}\right)\right]
\end{aligned}
$$

where $\mathcal{L}$ is the same as in (1), $b_{\mu} \equiv\left(\mu_{B} / N_{c}, 0\right)$, and, as usual, $q_{L}=\frac{1 \pm \gamma_{5}}{2} q$. As in [4], the following change of basis in the quark fields will yield a more suitable form for $S_{E}$ :

$Q_{L}=\xi q_{L} ; \quad Q_{R}=\xi^{\dagger} q_{R}$

with $\xi^{2}(x)=U(x)$. In this basis the euclidean action in Eq. (2) becomes:

$$
\begin{aligned}
S_{E} & =\int d^{4} x \bar{Q} \mathcal{D} Q \\
\mathcal{D} & =\gamma_{\mu}\left(\partial_{\mu}+\frac{i g}{2} \lambda^{a} G_{\mu}^{a}-b_{\mu}+\Gamma_{\mu}+\gamma_{5} \xi_{\mu}\right)-M \\
\Gamma_{\mu} & =\frac{1}{2}\left[\xi^{\dagger}\left(\partial_{\mu}+r_{\mu}\right) \xi+\xi\left(\partial_{\mu}+l_{\mu}\right) \xi^{\dagger}\right] \\
\xi_{\mu} & =\frac{1}{2}\left[\xi^{\dagger}\left(\partial_{\mu}+r_{\mu}\right) \xi-\xi\left(\partial_{\mu}+l_{\mu}\right) \xi^{\dagger}\right]
\end{aligned}
$$


where $v_{\mu}=\frac{1}{2}\left(r_{\mu}+l_{\mu}\right)$ and $a_{\mu}=\frac{1}{2}\left(r_{\mu}-l_{\mu}\right)$. Notice that $\Gamma_{\mu}$ and $\xi_{\mu}$ are both antihermitian. We see that in this basis $M$ gives rise to a mass term. The change of basis ( 3 ) induces a jacobian in the path integral measure that should be taken into account in the effective action. We shall now ignore this jacobian but we will study it later. We are only interested in the real part $\Gamma_{r}$ of the effective action $\Gamma_{q}$ defined in Eq. (2). The imaginary part gives rise to the WZW action [5], and will not be dealt with here. We have:

$\Gamma_{r} \equiv \operatorname{Re} \Gamma_{q}\left[\mu_{B}, v, a, U\right]=-\frac{1}{2} \log \operatorname{det} \mathcal{D}^{\dagger} \mathcal{D}$

The determinant of $\mathcal{D}^{\dagger} \mathcal{D}$ can be calculated using the $\zeta$-function regularization method $[17,18]$ :

$$
\begin{aligned}
& \log \operatorname{det} \mathcal{D}^{\dagger} \mathcal{D}=-\left.\frac{d}{d s} \zeta_{\mathcal{D}^{\dagger} \mathcal{D}}(s)\right|_{s=0} \\
& \zeta_{\mathcal{D}+\mathcal{D}}(s) \equiv \frac{\nu^{2 s}}{\Gamma(s)} \int_{0}^{\infty} d \tau \tau^{s-1} \\
& \quad \times \int d^{4} x \operatorname{Tr}\left\langle x\left|\exp \left(-\tau \mathcal{D}^{\dagger} \mathcal{D}\right)\right| x\right\rangle
\end{aligned}
$$

where $\nu$ is an arbitrary energy scale, the trace $\operatorname{Tr}$ is taken over Dirac, flavour and colour indices and $\Gamma(s)$ is the Euler Gamma function. From Eq. (4) we find:

$$
\begin{aligned}
& \mathcal{D}^{\dagger} \mathcal{D}=-\hat{\nabla}_{\mu} \hat{\nabla}_{\mu}+M^{2}-2 b^{2}+\hat{E} \\
& \hat{\nabla}_{\mu}=\partial_{\mu}+\frac{i g}{2} \lambda^{a} G_{\mu}^{a}+\Gamma_{\mu}+\gamma_{5} \xi_{\mu}-2 \sigma_{\mu \nu} b_{\nu} \\
& \quad \equiv \nabla_{\mu}-2 \sigma_{\mu \nu} b_{\nu} \\
& \hat{E}=-\sigma_{\mu \nu}\left[\nabla_{\mu}, \nabla_{\eta}\right]-2 M \gamma_{\mu} \gamma_{5} \xi_{\mu}+2 M \gamma_{\mu} b_{\mu}
\end{aligned}
$$

where $\sigma_{\mu \nu}=\left[\gamma_{\mu}, \gamma_{\nu}\right] / 4$. Notice that $\Gamma_{\mu}, \xi_{\mu}$ and $\hat{E}$ depend on $x$ and that they are not differential operators. The operator $\mathcal{D}^{\dagger} \mathcal{D}$ in Eq. (8) has the generic form of an elliptic second order differential operator. The following expansion:

$$
\begin{gathered}
\left\langle x\left|\exp \left(-\tau\left(\hat{E}-\hat{\nabla}_{\mu} \hat{\nabla}_{\mu}\right)\right)\right| x\right\rangle \\
=\sum_{n=0}^{\infty} H_{n}(x) \frac{\tau^{n}}{(4 \pi \tau)^{D / 2}}
\end{gathered}
$$

is called the Heat Kernel (HK) one (in the coincidence limit). The Seeley-de Witt coefficients $H_{n}(x)$ are explicitly known up to and including $H_{6}$ [18]. Here, $D$ is the euclidean space-time dimension. Notice that the expansion in powers of the momentum does not correspond directly with the HK expansion. We will treat first the case in which gluon fields are disregarded. Recall that in the $\mu_{B}=0$ case [4]: i) This approximation leads to the $o\left(p^{4}\right)$ effective lagrangian and to specific values for the coefficients of the various terms (with encouraging numerical agreement when compared to experimental data). ii) The $o\left(p^{2}\right)$ lagrangian is the non-linear sigma model, which, in turn, is used to relate the scale $M$ to $f_{\pi}$ and to derive the equations of motion for the $U$ field. iii) The gluon dependence can then be taken into account in the large $N_{c}$ limit, at a later stage, and it turns out to give rise to corrections to the various coefficients of the model. The same features are going to be met in our case, as we will see.

We now turn to calculate the effective action for $\mu_{B} \neq 0$, first without gluonic corrections. Then $\Gamma_{q}$ in Eq. (2) coincides with $\Gamma$ (as $\int[\tilde{d G}]=1$, by a suitable choice of the normalization constant embodied in $[\tilde{d G}])$. When the coupling of gluon fields to quarks are neglected, the chiral power counting is easier. The relevant coefficients in the HK expansion are given by [18]:

$$
\begin{aligned}
& H_{0}(x)=1 ; \quad H_{1}(x)=-\hat{E} \\
& H_{2}(x)=\frac{1}{2} \hat{E}^{2}-\frac{1}{6}\left[\hat{\nabla}_{\mu},\left[\hat{\nabla}_{\mu}, \hat{E}\right]\right]+\frac{1}{12} \hat{R}_{\mu y} \hat{R}_{\mu \nu} \\
& H_{3}(x)=-\frac{1}{6} \hat{E}^{3}+\frac{1}{12}\left(\left\{\hat{E},\left[\hat{\nabla}_{\mu},\left[\hat{\nabla}_{\mu}, \hat{E}\right]\right]\right\}\right. \\
& \quad+\left(\left[\hat{\nabla}_{\mu}, \hat{E}\right]\right)\left(\left[\hat{\nabla}_{\mu}, \hat{E}\right]\right)+o\left(p^{6}\right) \\
& H_{4}(x)=\frac{1}{24} \hat{E}^{4}+o\left(p^{6}\right)
\end{aligned}
$$

where:

$$
\begin{aligned}
\hat{R}_{\mu \nu} & \equiv\left[\hat{\nabla}_{\mu}, \hat{\nabla}_{\nu}\right] \\
& =\Gamma_{\mu \nu}+\gamma_{5} \xi_{\mu \nu}+\left[\xi_{\mu}, \xi_{\nu}\right]+4 b_{\alpha} b_{\beta}\left[\sigma_{\mu \alpha}, \sigma_{\nu \beta}\right] \\
\Gamma_{\mu \nu} & \equiv \partial_{\mu} \Gamma_{\nu}-\partial_{\nu} \Gamma_{\mu}+\left[\Gamma_{\mu}, \Gamma_{\nu}\right] \\
\xi_{\mu \nu} & \equiv d_{\mu} \xi_{\nu}-d_{\nu} \xi_{\mu} ; \quad d_{\mu} \equiv \partial_{\mu}+\left[\Gamma_{\mu}, \cdot\right]
\end{aligned}
$$

If the gluon fields are neglected, $\hat{R}_{\mu \nu}$ is $o\left(p^{2}\right), \hat{E}$ is $o(p)$, and then only the terms displayed in Eq. (10) contribute to the $o\left(p^{4}\right)$ lagrangian. Now, from (6), (7), (8) and (9) we have: 


$$
\begin{aligned}
\mathrm{G}_{r} & =\frac{1}{2} \frac{d}{d s} \frac{\nu^{2 s}}{\gamma(s)} \int_{0}^{\infty} d \tau \tau^{s-1} e^{-\tau\left(M^{2}-2 b^{2}\right)} \\
& \times\left.\int d^{4} x \operatorname{Tr} \sum_{n=0}^{\infty} \frac{H_{n}(x) \tau^{n}}{(4 \pi \tau)^{D / 2}}\right|_{s=0} \equiv \sum_{n=0}^{\infty} \mathrm{G}_{r}^{(n)}
\end{aligned}
$$

Notice that the condition $\mu_{B}^{2}<N_{c}^{2} M^{2} / 2$ appears naturally here and can be interpreted as what characterizes the maximum range of applicability of our approximation. The low density regime may then be defined as $\mu_{B}^{2} \ll M^{2}$. We obtain up to $o\left(p^{4}\right)$ :

$$
\begin{aligned}
& G_{r}^{(0)}=\frac{N_{c}}{16 \pi^{2}} \int d^{4} x \\
& \times \operatorname{tr}\left[\frac{\left(M^{2}-2 b^{2}\right)^{2}}{4}\left(\frac{3}{2}+\log \frac{\nu^{2}}{\left(M^{2}-2 b^{2}\right)}\right)\right] \\
& G_{r}^{(2)}=\frac{N_{c}}{32 \pi^{2}} \log \frac{\nu^{2}}{\left(M^{2}-2 b^{2}\right)} \int d^{4} x \\
& \times \operatorname{tr}\left[-\frac{2}{3}\left(\left(\mathrm{G}_{\mu \nu}+\left[\xi_{\mu}, \xi_{\nu}\right]\right)^{2}+\xi_{\mu \nu}^{2}\right)-8 M^{2} \xi^{2}\right. \\
&\left.+8 M^{2} b^{2}-8 b^{4}\right] \\
& \mathrm{G}_{r}^{(3)}=\frac{N_{c}}{32 \pi^{2}} \frac{M^{2}}{M^{2}-2 b^{2}} \int d^{4} x \\
& \times \operatorname{tr}\left[8 \xi_{\mu} \xi_{\alpha}\left(\mathrm{G}_{\mu \alpha}+\left[\xi_{\mu}, \xi_{\alpha}\right]\right)-\frac{4}{3} \xi_{\beta} d^{2} \xi_{\beta}\right. \\
&- \frac{8}{3} \xi_{\alpha} \xi_{\beta} \xi_{\alpha} \xi_{\beta}-\frac{8}{3} \xi^{2} \xi^{2}-16 \xi_{\alpha \beta} \xi_{\alpha} b_{\beta} \\
&- 8 \epsilon_{\mu \nu \alpha \beta} b_{\mu} \xi_{\nu}\left(\mathrm{G}_{\alpha \beta}+\left[\xi_{\alpha}, \xi_{\beta}\right]\right) \\
&+\left.\frac{32}{3} b_{\mu}\left(d_{\alpha} \xi_{\mu}\right) \xi_{\alpha}+32 b^{2} \xi^{2}-\frac{32}{3} b_{\alpha} b_{\beta} \xi_{\alpha} \xi_{\beta}\right] \\
& \mathrm{G}_{r}^{(4)}=\frac{N_{c}}{32 \pi^{2}} \frac{M^{4}}{\left(M^{2}-2 b^{2}\right)^{2}} \int d^{4} x \\
& \quad \times \operatorname{tr}\left[\frac{8}{3}\left(2 \xi^{2} \xi^{2}-\xi_{\alpha} \xi_{\beta} \xi_{\alpha} \xi_{\beta}\right)+\frac{32}{3} \epsilon_{\mu \nu \alpha \beta} b_{\mu} \xi_{\nu} \xi_{\alpha} \xi_{\beta}\right. \\
&\left.\quad-16 b^{2} \xi^{2}+\frac{32}{3} b_{\alpha} b_{\beta} \xi_{\alpha} \xi_{\beta}+\frac{8}{3} b^{4}\right]
\end{aligned}
$$

where the trace tr runs only over flavour indices. In Eq. (13), terms that are total $d_{\alpha}$ derivatives have been omitted.

The final step will lead from Eq. (13) to the effective action in terms of GB fields $U(x)$ and $F_{\mu \nu}^{L}=$ $\partial_{\mu} l_{\nu}-\partial_{\nu} l_{\mu}+\left[l_{\mu}, l_{\nu}\right], F_{\mu \nu}^{R}=\partial_{\mu} r_{\nu}-\partial_{\nu} r_{\mu}+\left[r_{\mu}, r_{\nu}\right]$. This can be done from the definitions in Eqs. (5) and (11), by using properties given in [4]. In addition, since we treat $U(x)$ as a classical field, it satisfies the equations of motion in the chiral limit:

$$
\left(D^{2} U\right) U^{\dagger}-U\left(D^{2} U^{\dagger}\right)=0
$$

where the covariant derivatives acting over $U$ fields are given by:

$$
\begin{aligned}
& D_{\mu} U=\partial_{\mu} U+r_{\mu} U-U l_{\mu} \\
& D_{\mu} U^{\dagger}=\partial_{\mu} U^{\dagger}-U^{\dagger} r_{\mu}+l_{\mu} U^{\dagger}
\end{aligned}
$$

Eqs. (14) follow from the $o\left(p^{2}\right)$ effective lagrangian, which is the non-linear sigma model. Then, it is consistent to use them in the $o\left(p^{4}\right)$ lagrangian. These equations remain as they stand when $\mu_{B} 30$ is included, because there are no terms at $o\left(p^{2}\right)$ depending on the $U$ field and $\mu_{B}$ simultaneously. To be consistent, as $\mu_{B}$ is of order $o(p)$ and $o\left(p^{6}\right)$ terms have been neglected, we have to expand the constant factors appearing in Eq. (13) up to $o\left(\mu_{B}^{4}\right)$ in $\mathrm{G}_{r}^{(0)}$ and up to $o\left(\mu_{B}^{2}\right)$ in the second and third terms in $\mathrm{G}_{r}^{(2)}$. In the remaining terms, those factors have to be taken at $\mu_{B}=0$.

Regarding the various $\mu_{B}$ dependent terms appearing in Eq. (13) we notice that the terms $b^{2} \xi^{2}$ and $b_{\alpha} b_{\beta} \xi_{\alpha} \xi_{\beta}$ would give rise in the effective action to $\mu_{B}^{2} D_{\mu} U D_{\mu} U^{\dagger}$ (which, in turn, would imply a renormalization of $f_{\pi}$, as it will appear in (16)) and to $\mu_{B}^{2} D_{0} U D_{0} U^{\dagger}$, respectively. One of the most surprising features of our computation is the fact that the coefficients multiplying both terms vanish exactly, and independently, which can be seen directly from Eq. (13) by expanding into powers of $\mu_{B}$. In addition, the term $\left(d_{\alpha} \xi_{\mu}\right) \xi_{\alpha}$ vanishes identically in the chiral limit. Thus, in the absence of gluon fields we finally arrive to the following finite density euclidean effective action at $o\left(p^{4}\right)$ :

$$
\begin{aligned}
\mathrm{G}_{r} & {\left[U, \mu_{B}, F^{L}, F^{R}\right]=\mathrm{G}_{r}^{c}\left[\mu_{B}\right] } \\
& +\int d^{4} x \operatorname{tr}\left[\frac{1}{4} f_{\pi}^{2} D_{\mu} U D_{\mu} U^{\dagger}\right. \\
& +L_{2}\left(D_{\mu} U^{\dagger} D_{\nu} U D_{\mu} U^{\dagger} D_{\nu} U\right) \\
& +H_{1}\left(F_{\mu \nu}^{R} F_{\mu \nu}^{R}+F_{\mu \nu}^{L} F_{\mu \nu}^{L}\right) \\
& +L_{9}\left(F_{\mu \nu}^{R} D_{\mu} U D_{\nu} U^{\dagger}+F_{\mu \nu}^{L} D_{\mu} U^{\dagger} D_{\nu} U\right) \\
& +L_{10} U^{\dagger} F_{\mu \nu}^{R} U F_{\mu \nu}^{L} \\
& -\frac{\mu_{B}}{16 \pi^{2}} \epsilon_{i j k}\left(F_{i j}^{L} U^{\dagger} D_{k} U+F_{i j}^{R} D_{k} U U^{\dagger}\right) \\
& -\frac{\mu_{B}}{8 \pi^{2}}\left(D_{i} U U^{\dagger} F_{i 0}^{R}-U^{\dagger} D_{i} U F_{i 0}^{L}\right) \\
& \left.+\frac{\mu_{B}}{24 \pi^{2}} \epsilon_{i j k} U^{\dagger} D_{i} U U^{\dagger} D_{j} U U^{\dagger} D_{k} U\right]
\end{aligned}
$$


The term $\Gamma_{r}^{c}\left[\mu_{B}\right]$ (which would represent a shift in the free energy density) is:

$$
\begin{gathered}
\Gamma_{r}^{c}\left[\mu_{B}\right]=\frac{N_{c}}{32 \pi^{2}} \int d^{4} x \operatorname{tr}\left[\frac{M^{4}}{2}\left(\frac{3}{2}+\log \frac{\nu^{2}}{M^{2}}\right)\right. \\
+2 \frac{\mu_{B}^{2}}{N_{c}^{2}} M^{2}\left(3 \log \frac{\nu^{2}}{M^{2}}-1\right) \\
\left.+\frac{\mu_{B}^{4}}{N_{c}^{4}}\left(\frac{62}{3}-6 \log \frac{\nu^{2}}{M^{2}}\right)\right]
\end{gathered}
$$

The $\mu_{B}=0$ part in Eq. (16) is the $o\left(p^{2}\right)$ plus $o\left(p^{4}\right)$ lagrangian already obtained in [1]. The various coefficients appearing in it coincide with those obtained in previous $\mu_{B}=0$ calculations [2-4], as they should. For instance, $f_{\pi}$ is related to $M$ through $f_{\pi}^{2}=\frac{N_{c}}{4 \pi^{2}} M^{2} \log \nu^{2} / M^{2}$. A typical value for $M$ giving good numerical agreement for the coefficients is $M \simeq 320 \mathrm{MeV}$ [4]. Regarding the terms appearing in the complete $o\left(p^{4}\right)$ lagrangian in [1] that are not displayed in Eq. (16), notice that the corresponding coefficients vanish in the large $N_{c}$ limit.

We shall now treat the jacobian of the change of basis given in Eq. (3). It can be computed following the methods used by Fujikawa [19] in the calculation of anomalies. Since the $\mathcal{D}$ operator is not hermitian, we choose to regularize the jacobian as it was done in the sccond reference of [19]. With this method, the $Q$ fields are expanded in terms of eigenfunctions of $H \equiv \mathcal{D}^{\dagger} \mathcal{D}$ and the $\bar{Q}$ ones in terms of those of $\bar{H} \equiv \mathcal{D} \mathcal{D}^{\dagger}$. Notice that $H\left(b_{\mu}, \Gamma_{\mu}, \xi_{\mu}, M\right)=$ $\bar{H}\left(-b_{\mu}, \Gamma_{\mu},-\xi_{\mu},-M\right)$. Then, the HK expansion is used with the coefficients given in Eq. (10) and only $\mathrm{H}_{2}$ survives now in the regularization procedure. We have checked that the jacobian regulated in this way is indeed independent of $\mu_{B}$, which does agree with previous analysis [20]. Furthermore, the jacobian itself gives contributions only to the WZW action $[3,5,17]$. Consequently, it is irrelevant for our purposes and will not be taken into account here.

We now turn to comment about the different $\mu_{B^{-}}$ dependent terms obtained in the effective lagrangian displayed in Eq. (16). Based only upon symmetry arguments, one could have asked what would be the most general set of operators that have all the symmetries of the original QCD lagrangian, in the actual $\mu_{B} \neq 0$ case. Notice that this point of view tells nothing about the precise values of the coefficients of the different operators. Obviously, if the symmetries of the $\mu_{B} \neq 0$ lagrangian were the same as those for the $\mu_{B}=0$ one, the only possible $\mu_{B}$ dependencies would appear through renormalizations of the various coefficients of the operators in the original lagrangian. Then, up to the order $o\left(p^{4}\right)$ considered here, the only dependencies would have been in the renormalization of $f_{\pi}$ previously mentioned. However, the chemical potential term in Eq. (1) does not respect two properties that were valid for $\mu_{B}=0$, namely, charge conjugation (C) symmetry and Euclidean covariance. The breaking of the latter covariance is due to the inclusion of the constant four-vector $b_{\mu}$. Since the only Euclidcan four-vcctor appearing in the $\mu_{B}=0$ lagrangian is $D_{\mu}$, we could get different operators by replacing $D_{\mu}$ vectors by $b_{\mu}$, in suitable combinations so as to preserve both chiral $S U_{L}\left(N_{f}\right) \times S U_{R}\left(N_{f}\right)$ symmetry and parity $(P)$ invariance. This is what happens with the second $\mu_{B}$-dependent term in Eq. (16), which can be obtained in this way from the $L_{9}$ term in the original $\mu_{B}=0$ lagrangian. Another operator with these features would be $\mu_{B}^{2} D_{0} U D_{0} U^{\dagger}$, which comes from the $L_{2}$ piece (and which does not appear in (16), as explained above).

Other types of operators would be those violating $C$ but preserving $P$ and chiral invariance. Two operators of this type are the first and the third $\mu_{B}$ dependent ones in Eq. (16). The first one depends on the external sources (and on $U$ ), while the third one depends only on the $U$ field. Notice that these two terms, although real, are anomalous, that is, they are odd under the spatial parity operation $P_{0}:(t, x) \rightarrow(t,-x)$ for any four-vector $(t, \boldsymbol{x})$. Of course, they are also odd under the simultaneous operation $U \leftrightarrow U^{\dagger}, L \leftrightarrow R$. The parity operation $P$ is the product of $P_{0}$ and the latter operation, so these terms are $P$ invariant, as they should. However, as they are anomalous, when $U$ is expanded in terms of $\pi^{a}$ fields, only odd powers of $\pi^{a}$ will appear. Then, they could in principle contribute to anomalous processes (in $\chi \mathrm{PT}$ ), like $\pi^{0} \rightarrow \gamma \gamma$ at $\mu_{B} \neq 0$ (a process studied previously in [21] at finite temperature but for $\mu_{B}=0$ ). From the point of view of building up $\mu_{B} \neq 0$ operators commented before, one can regard these anomalous terms as coming from the gauged WZW term $[14,15]$ when the external fields are replaced by $b_{\mu}$. We remark that when external fields are not considered (so that $l_{\mu}=r_{\mu}=0$ ), the last term in Eq. (16) turns out to be the baryon 
number, written now in terms of $U$ fields. Recall that, as discussed in [15], when the WZW anomalous action is considered one can cast the baryon number current as:

$J_{\mu}^{B}=\frac{1}{24 \pi^{2}} \epsilon_{\mu \nu \alpha \beta} U^{\dagger} \partial_{\nu} U U^{\dagger} \partial_{\alpha} U U^{\dagger} \partial_{\beta} U$

Then we conclude that in our effective action in Eq. (16), a term of the form $\mu_{B} N_{B}$ has been derived, $N_{B}$ being the baryon number with the correct normalization factor. This term is indeed a topological one, because $N_{B}$ turns out to be the winding number of mappings $U(\boldsymbol{x})$ from $S^{3}$ into $S U\left(N_{f}\right)[15,17]$.

Next, we will take into account the coupling of quarks to gluon fields. In the large $N_{c}$ limit, the leading approximation in the cumulant expansion [4]:

$$
\langle\exp -X\rangle \simeq \exp -\langle X\rangle ; \quad\langle X\rangle=\int[\tilde{d G}] X
$$

appears to be correct for $\mu_{B} \neq 0$ as it certainly was for $\mu_{B}=0$ [4]. That is, we replace the gluonic invariants appearing in the calculation by their QCD expectation values. The first possible gluonic correction will be then proportional to the gluon condensate $\left\langle\alpha / \pi G_{\mu \nu}^{a} G_{\mu \nu}^{a}\right\rangle$ (with $\alpha=g^{2} / 4 \pi$ ), which is known to have a non-perturbative part [22]. Its phenomenological value has many uncertainties. The value for $M \simeq 320 \mathrm{MeV}$ is consistent with $\left\langle\alpha / \pi G_{\mu \nu}^{a} G_{\mu \nu}^{a}\right\rangle \simeq$ $[410 \mathrm{MeV}]^{4}[4,23]$. Here, we will calculate the first gluonic corrections to the $\mu_{B}$ dependent terms which would vanish exactly if gluon fields were neglected (see above). We will consider only the corrections proportional to the gluon condensate. It is not easy to estimate the order of magnitude of the terms we are neglecting. However, recall that when only gluon condensate contributions (for $\mu_{B}=0$ ) were considered in [4], the numerical agreement for the coefficients became rather remarkable, the results being always better than the ones obtained without gluon fields. Furthermore, regarding the gluonic corrections to $f_{\pi}$, the next ones, proportional to the triple gluon condensate $\langle G G G\rangle$, have been computed for $\mu_{B}=0$ in [4] and found to be subdominant.

When gluonic corrections are included as indicated above, the calculation becomes very much involved, even if we concentrate only in the terms we are interested in. Recall that $\hat{R}_{\mu \nu}$ defined in Eq. (11) gets modified as $\hat{R}_{\mu \nu} \rightarrow \hat{R}_{\mu \nu}+G_{\mu \nu}$, with $G_{\mu \nu}=\frac{i g}{2} \lambda^{a} G_{\mu \nu}^{a}$. The new term is $o(1)$ in the chiral power counting. The same happens with $\hat{E} \rightarrow \hat{E}-\sigma_{\mu \nu} G_{\mu \nu}$. Consequently, we have to treat $H_{4}$ in order to find a term proportional to $G G \xi^{2}$ (the non-linear sigma model piece), which is $o\left(p^{2}\right)$. This term has been analyzed for $\mu_{B}=0$ in [4]. When trying to calculate the $\mu_{B}$ corrections commented above, we have to look for contributions proportional to $\mu_{B}^{2} G G \xi^{2}$. Since these terms are $o\left(p^{4}\right)$ we have now to go beyond $H_{4}$. In fact, for $\mu_{B} \neq 0$ we find the searched contributions in the $H_{5}$ and $H_{6}$ coefficients [18]. Finally we arrive at the following result. When gluonic corrections are included, the real part of the effective action $\Gamma$ in Eq. (2) is given by $\operatorname{Re} \Gamma=\Gamma_{r}+\Gamma_{G}, \Gamma_{r}$ being given in Eq. (16) and:

$$
\begin{aligned}
\Gamma_{G} & =\frac{1}{32} \frac{\left\langle\alpha / \pi G_{\mu \nu}^{a} G_{\mu \nu}^{a}\right\rangle}{M^{4}}\left(\frac{M^{2}}{3} D_{\mu} U D_{\mu} U^{\dagger}\right. \\
& \left.+\frac{364}{135} \frac{\mu_{B}^{2}}{N_{c}^{2}} D_{\mu} U D_{\mu} U^{\dagger}-\frac{232}{135} \frac{\mu_{B}^{2}}{N_{c}^{2}} D_{0} U D_{0} U^{\dagger}\right)
\end{aligned}
$$

The first term in the above equation gives the first gluonic correction to $f_{\pi}^{2}$ for $\mu_{B}=0$, calculated in [4]. If $U$ is again expanded in terms of $\pi^{a}$ fields, the $\mu_{B}$ dependent contributions in Eq. (20) give rise, at the lowest order $o\left(\pi^{2}\right)$, to a $\mu_{B}$-dependent modification of the propagator for the $\pi^{a}$ fields. Thus, this effect would appear, via $\chi \mathrm{P}$, in chiral loops, now at nonzero $\mu_{B}$. This correction, in momentum space $\left(p \equiv\left(p_{0}, p\right)\right)$ would modify the $p_{0}^{2}$ and the $p^{2}$ dependencies of the propagator by different amounts, thereby reflecting the loss of covariance previously mentioned. The $\mu_{B}$ dependent terms obtained in Eq. (20) can give rise, via PCAC, to density corrections to the physical value of $f_{\pi}$ (which for $\mu_{B}=0$ and to lowest order in $\pi$ fields, coincides with the $f_{\pi}$ appearing in the lagrangian [17]). The absence of these terms when gluon corrections are not considered (see above) seems to agree with the results in [9], in which $f_{\pi}$ scales with density due to the trace anomaly of QCD, proportional to $G_{\mu \nu}^{a} G_{\mu \nu}^{a}$ in the chiral limit. In fact, it can be seen that the $\mu_{B}$-dependent terms in (16) do not contribute to the physical valuc of $f_{\pi}$.

To summarize: 1) We have obtained the real part of the effective action at nonzero $\mu_{B}$, up to $o\left(p^{4}\right)$ in the large $N_{c}$ limit. 2) Precise values for the coefficients multiplying every $\mu_{B}$-dependent term have been obtained. 3) A piece $\mu_{B} N_{B}$ arises, $N_{B}$ being the topo- 
logical baryon number, which physically corresponds to baryon number conservation. 4) A term contributing to anomalous processes at finite $\mu_{B}$ has also been obtained. 5) When gluonic corrections proportional to the gluon condensate are included, two terms quadratic in $\mu_{B}$ and in derivatives of the $U$ field are also found, with their corresponding cocfficients. The lagrangian obtained here could be an starting point for the study of density effects on: i) physical observables, in the low density regime, using for instance $\chi \mathrm{PT}$, ii) Thermodynamical properties of hadrons near the chiral phase transition. The analysis of i) and ii) lies beyond the scope of this work.

We thank Prof. A. Dobado for useful information. The financial supports of CICYT (Proyecto AEN93-0776), Spain, and Human Capital and Mobility Programme, European Commission (Contract ERBCHRXCT940423), Brussels, are acknowledged.

\section{References}

[1] J. Gasser and H. Leutwyler, Ann. Phys. (N.Y.) 158 (1984); Nucl. Phys B 250 (1985) 465, 517, 539.

[2] A.A. Andrianov and L. Bonora, Nucl. Phys. B 233 (1984) 232

A.A. Andrianov, Phys. Lett. B 157 (1985) 425.

[3] N.I. Karchev and A.A. Slavnov, Theor. Mat. Phys. 65 (1985) 192.
[4] D. Espriu, E. de Rafael and J. Taron, Nucl. Phys. B 345 (1990) 22.

[5] J. Bijnens, Nucl. Phys. B 367 (1991) 709.

[6] A. Schenk, Phys. Rev. D 47 (1993) 5138.

[7] P. Gerber and H. Leutwyler, Nucl. Phys. B 321 (1989) 387.

[8] A. Barducci, R. Casalbuoni, S. de Curtis, R. Gatto and G. Pettini, Phys. Rev. D 41 (1990) 1610.

[9] G.E. Brown and M. Rho, Phys. Rev. Lett. 66 (1991) 2720; C. Adami and G.E. Brown, Phys. Rep. 234 (1993) 1, and references therein.

[10] V. Bernard, U.G. Meissner and I. Zahed, Phys. Rev. D 36 (1987) 819.

[11] C.W. Bernard, Phys. Rev. D 9 (1974) 3312.

[12] P.D. Morley and M.B. Kislinger, Phys. Rep. 51 (1979) 63.

[13] N.P. Landsman and Ch.G. Van Weert, Phys. Rep. 145 (1987) 1941.

[14] J. Wess and B. Zumino, Phys. Lett. B 37 (1971) 95; L. Alvarez-Gaumé and P. Ginsparg, Ann. Phys. 161 (1985) 423.

[15] E. Witten, Nucl. Phys. B 223 (1983) 422, 433.

[16] A. Manohar and H. Georgi, Nucl. Phys. B 234 (1984) 189.

[17] J. Donogue, E. Golowich and B.R. Holstein, Dynamics of the standard model, Cambridge University Press, 1994.

[18] R.D. Ball, Phys. Rep. 182 (1989) 1.

[19] K. Fujikawa, Phys. Rev. D 21 (1980) 2848; D 29 (1984) 285.

[20] A. Gómez Nicola and R.F. Alvarez-Estrada, Int. J. Mod. Phys. A 9 (1994) 1423.

[21] R.F. Alvarez-Estrada, A. Dobado and A. Gómez Nicola, Phys. Lett. B 324 (1994) 345.

[22] M.A. Shifman, A.I. Vainshtein and V.I. Zakharov, Nucl. Phys. B 147 (1979) 385, 448.

[23] R.A. Bertlmann, C.A. Dominguez, M. Loewe, M. Perrottet and E. de Rafael, Z. Phys. C 39 (1988) 231. 